\title{
Application of Fluorinated Alkyl Acrylate to Denture Base Resin Influence of Carbon Chain Length of Fluorinated Alkyl Acrylate on Bacterial Adherence
}

\author{
Masato Kobayashi, ${ }^{1}$ Toshio Kubota, ${ }^{2}$ and Junichi Mega ${ }^{3,4}$ \\ ${ }^{1}$ Nihon University Graduate School of Dentistry at Matsudo, Dentistry for the Disabled, Matsudo, Chiba 271-8587, \\ Japan \\ ${ }^{2}$ Department of Biomolecular Functional Engineering, Ibaraki University, Hitachi, Ibaraki 316-8511, Japan \\ ${ }^{3}$ Department of Dentistry for the Disabled, and ${ }^{4}$ Research Institute of Oral Science, Nihon University School of \\ Dentistry at Matsudo, Matsudo, Chiba 271-8587, Japan
}

Correspondence to :

Masato Kobayashi

E-mail : kobayashi.masato@ nihon-

u.ac.jp

Keywords :

denture base resin, fluorinated alkyl acrylate, hydrophobicity, Candida albicans

\begin{abstract}
This study examined the influence of carbon chain length of fluorinated alkyl acrylate on bacterial adhesion to the surface of test specimens. Perfluorobutylethyl acrylate $(\mathrm{C} 4 \mathrm{~F})$, perfluorohexylethyl acrylate $(\mathrm{C} 6 \mathrm{~F})$, perfluorooctylethyl acrylate $(\mathrm{C} 8 \mathrm{~F})$, and perfluorodecylethyl acrylate $(\mathrm{C} 10 \mathrm{~F})$ were used as fluorinated alkyl acrylate monomers. All specimens with the fluoro-monomer added exhibited significantly higher water-shedding and oil-shedding surface characteristics when compared to control (MMA/PMMA). Furthermore, contact angle for droplets of water and dodecane on the fluoromonomer-added specimens increased with carbon chain length of the perfluoroalkyl groups. Bacterial adhesion was significantly suppressed on the fluoromonomer -added specimens, both the saliva-uncoated and -coated specimens. In addition, the number of Candida albicans adhering to both the salivauncoated and -coated fluoromonomer-added specimens decreased with an increase in perfluoroalkyl carbon chain length. The results suggest that the application of fluorinated alkyl acrylate to denture base resin can modify the surface characteristics, and that fluorinated alkyl acrylate with long carbon chains of $\mathrm{C} 8 \mathrm{~F}$ and $\mathrm{C} 10 \mathrm{~F}$ successfully inhibit bacterial adherence to the resin surface.
\end{abstract}

\section{Introduction}

Demographic data indicate an increasing proportion of elderly in the population and a lengthening life expectancy. Elderly adults are retaining their natural teeth longer, and the first removable dentures are being obtained later in life. The use of dentures is beneficial to physical as well as oral function (1). However, plaque adhering to the denture surface can cause regional inflammation in oral mucosa, such as denture stomatitis, and systemic disease, such as bacterial pneumonia and infectious endocarditis (2). Yoneyama et al. suggested that oral care might prevent respiration infections in elderly adults because improved oral hygiene in the elderly reduced the duration of pneumonia-induced fever (3). Denture plaque can be removed with a brush or denture cleaner. However, it can be difficult for ill older adults and people with disabilities to clean their artificial dentures. Inhibiting plaque formation on denture surfaces through the development of a new denture base material that prevents microbial adhesion could solve this problem.

Hayashi et al (4). suggested that the surface characteristics of denture base resin could be improved markedly by adding perfluorooctyl ethyl acrylate to the filling polymer. Previously, we examined the influence of carbon chain length of fluorinated alkyl acrylate on mechanical properties (5). In this study, 
we examine the influence of carbon chain length of fluorinated alkyl acrylate on the surface properties and surface bacterial adhesion using fluorinated alkyl acrylate monomers such as perfluorobutyl ethyl acrylate $(\mathrm{C} 4 \mathrm{~F})$, perfluorohexyl ethyl acrylate $(\mathrm{C} 6 \mathrm{~F})$, perfluorooctyl ethyl acrylate $(\mathrm{C} 8 \mathrm{~F})$, and perfluorodecyl ethyl acrylate (C10F).

\section{Materials and Methods}

\section{Preparation of Polymer beads}

Perfluorobutyl ethyl acrylate $(\mathrm{C} 4 \mathrm{~F})$, perfluorohexyl ethyl acrylate $(\mathrm{C} 6 \mathrm{~F})$, perfluorooctyl ethyl acrylate $(\mathrm{C} 8 \mathrm{~F})$, and perfluorodecyl ethyl acrylate $(\mathrm{C} 10 \mathrm{~F})$ were purchased from Daikin Chemicals Sales Company, Ltd. and used as the fluoromonomers. Methyl methacrylate (MMA) (Kanto Chemical Company, Ltd.) was used as a control and base monomer. According to the method of Kim et al (6), spherical beads were made by suspension polymerization. The results of a preliminary experiment showed that beads with even diameters could be collected using a monomer weight ratio of $25 \mathrm{wt} \%$ fluoromonomer and $75 \mathrm{wt} \%$ MMA, although spherical beads could not be collected at monomer weight ratios greater than $25 \mathrm{wt} \%$ (7). Control beads were made in the same manner using only MMA.

\section{Preparation of Control and Fluoromonomer-added specimen}

Test specimens were made using the method described by Hayashi et al (5). Briefly, paraffin wax was attached to the four sides of an acryl board (size : $20 \mathrm{~mm} \times 50 \mathrm{~mm}$, thickness : $2 \mathrm{~mm}$ ) in the shape of a truncated pyramid, and the resulting acryl board was embedded in plaster while being sandwiched by slide glass to prepare a counter-die. The liquid portion was prepared at a monomer weight ratio of 25 wt\% fluoromonomer and $75 \mathrm{wt} \%$ MMA. The polymer to liquid ratio (wt/wt) was 2: 1 for both the control (MMA) and the fluoromonomer-added specimens (C4FA, C6FA, C8FA, and C10FA). Then, 1.5 wt $\% \alpha^{-} \alpha^{\prime}$-azobisisobutyronitrile (AIBN) was added to initiate polymerization. These ingredients were mixed in a conventional manner, and cast in the counter-die to allow polymerization to take place at $70{ }^{\circ} \mathrm{C}$ for $6 \mathrm{hr}$ and then at $100{ }^{\circ} \mathrm{C}$ for $0.8 \mathrm{hr}$. From the center of the resulting resin plate, four test specimens $(10 \times 20 \mathrm{~mm})$ were cut. Two of the four specimens were used to prepare polished specimens as follows. The two specimens were polished using \#1200, \# 2000, \# 4000, and \# 8000 water-resistant sandpapers (Sumitomo 3M), and the surface of each piece was polished using a lathe and then cleaned ultrasonically with a rouge cleaner. The other two specimens were cleaned ultrasonically in distilled water for testing as unpolished specimens.

\section{Measurement of Contact angle}

Contact angles were measured by the sessile drop method using a contact angle analyzer (KYOWA Contact Angle Mater CA-D, Kyowa Science Co., Ltd.) on the polished specimen surfaces. Droplets of distilled water (D.W., $6 \mu$ l) or dodecane (Sigma-Aldrich, $6 \mu \mathrm{l}$ ) were placed at two different locations on test specimens to measure the contact angles (8).

\section{Analysis of Microbial Adhesion}

Candida albicans strain ATCC90029 was used to examine microbial adhesion. Microbial adhesion to saliva-uncoated and -coated specimens was analyzed according to the methods of Otake et al (9). and Kohinata (10), respectively. Briefly, unstimulated whole saliva samples were collected from a healthy male donor to eliminate sample variation. The saliva was clarified by centrifugation at $10,000 \times \mathrm{g}$ for 10 min. Saliva samples were stored at $-80{ }^{\circ} \mathrm{C}$ prior to use. A hole was made at the edge of the polished and unpolished specimen surfaces, which were then immersed in distilled water for a week (saliva-uncoated specimen). The polished surface specimens, which were immersed in distilled water for one week, were incubated in $3 \mathrm{ml}$ saliva with agitation at $37^{\circ} \mathrm{C}$ for 1 hr (saliva-coated specimen).

A test solution was prepared by culturing C. albicans in liquid culture medium according to conventional methods. C. albicans was subcultured in liquid BHI (Difco ${ }^{\circledR}$ ) culture medium and incubated with agitation at $37^{\circ} \mathrm{C}$ for $24 \mathrm{hr}$ to achieve the middle of 
the logarithmic growth phase. The resulting culture was centrifuged at 3,000 rpm for $10 \mathrm{~min}$. After washing the centrifuged C. albicans three times with phosphate-buffered saline (PBS), C. albicans was incubated in $2 \mathrm{ml}$ saliva with agitation at 120 shakes per minute for $1 \mathrm{hr}$ and centrifuged with PBS. A sonic disrupter (ultrasonic cell disrupter; Ohtake Seisakusho ; sound output: $20 \mathrm{kHz}, 50 \mathrm{~W}, 30 \mathrm{sec}$ ) was used to disperse $C$. albicans, and PBS was added to adjust microbial concentration to approximately $1 \times 10^{8}$ cells $/ \mathrm{ml}$. Test specimens were suspended by stainless steel wires into $3 \mathrm{ml}$ of the test solutions, followed by incubation with agitation for $1 \mathrm{hr}$ at 120 shakes per minute. After removing excess test solution by washing, the resin specimens were stained using $0.01 \%$ crystal violet for one minute and rinsed with D.W. After drying the specimens, the numbers of adhered C. albicans were counted using a digital high fidelity microscope (VH-8000, Keyence Cooperation) at $\times 400$ magnification.

\section{Statistical Analysis}

The data were analyzed for statistical differences between control and experimental groups using oneway ANOVA, two-way ANOVA, and Tukey's test for multiple comparisons (11). Statistical calculations were performed using STATISTICA (Stat Sof $\left.t^{\circledR}\right)$. The correlation between the contact angle for water and the number of adhered C. albicans was determined using Pearson's correlation coefficient (12).

\section{Results}

\section{Contact Angle}

Tables 1 and 2 show the contact angles for water and dodecane, respectively. Contact angles for water on $\mathrm{C} 4 \mathrm{FA}, \mathrm{C} 6 \mathrm{FA}, \mathrm{C} 8 \mathrm{FA}$, and $\mathrm{C} 10 \mathrm{FA}$ were $88.5^{\circ}, 90.0^{\circ}$, $90.5^{\circ}$, and $91.2^{\circ}$, respectively; contact angle for the control was $71.9^{\circ}$. Contact angles of oil on C4FA, $\mathrm{C} 6 \mathrm{FA}, \mathrm{C} 8 \mathrm{FA}$, and $\mathrm{C} 10 \mathrm{FA}$ were $22.1^{\circ}, 28.6^{\circ}, 31.9^{\circ}$, and $30.8^{\circ}$, respectively ; contact angle for the control was $10.1^{\circ}$. All fluoromonomer-added specimens possessed significantly greater water-shedding and oil-shedding abilities. The contact angle of C4FA for water was lower than those of $\mathrm{C} 8 \mathrm{FA}$ and $\mathrm{C} 10 \mathrm{FA}$. Furthermore, the contact angle of $\mathrm{C} 4 \mathrm{FA}$ for dodecane was lower than those of $\mathrm{C} 6 \mathrm{FA}, \mathrm{C} 8 \mathrm{FA}$, and $\mathrm{C} 10 \mathrm{FA}$.

Table 1. Contact angle for water

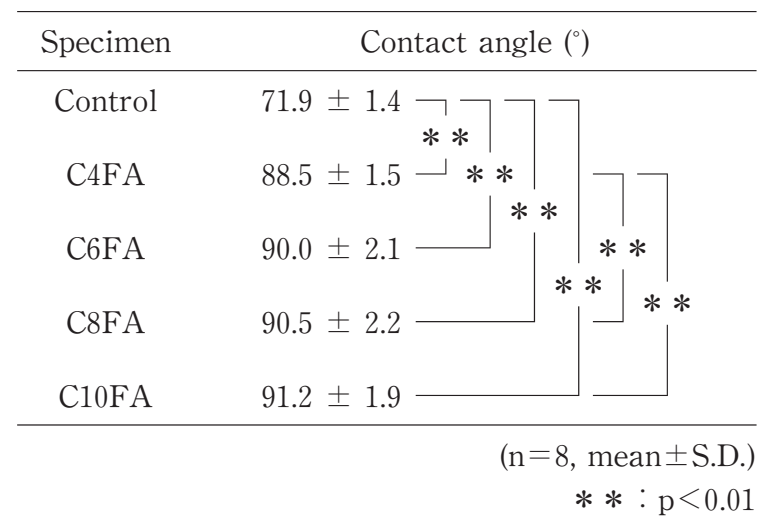

Table 2. Contact angle for dodecane

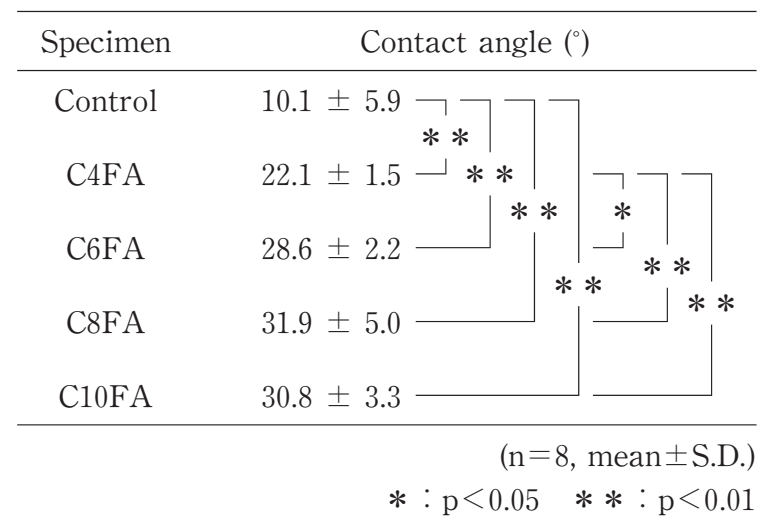

Adherence of C. albicans organisms to saliva-uncoated specimen surfaces

Fig. 1. shows the numbers of $C$. albicans organisms adhered to saliva-uncoated specimens. The numbers

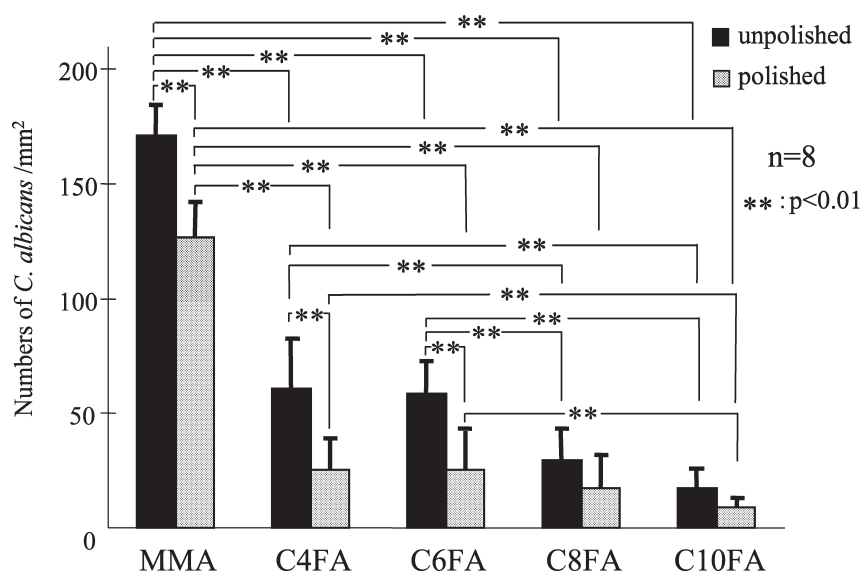

Fig. 1. Adherence of C. albicans to saliva-uncoated specimen 
adhered to unpolished surfaces of $\mathrm{C} 4 \mathrm{FA}, \mathrm{C} 6 \mathrm{FA}$, C8FA, and C10FA were 60.9, 58.8, 29.4, and 17.5 cells/ $\mathrm{mm}^{2}$, respectively, while the number for the control was 171.2 cells $/ \mathrm{mm}^{2}$. In contrast, the numbers of $C$. albicans organisms adhered to polished C4FA, C6FA, C8FA, and C10FA were 25.6, 25.4, 17.6, and 9.0 cells/ $\mathrm{mm}^{2}$, respectively, while that of the control was 126.5 cells $/ \mathrm{mm}^{2}$. The numbers of C. albicans organisms adhered to unpolished C4FA and C6FA were greater than those found for polished $\mathrm{C} 4 \mathrm{FA}$ and $\mathrm{C} 6 \mathrm{FA}$. The numbers adhered to specimens containing fluorinated alkyl acrylate were significantly less than that of the control. Furthermore, the numbers of $C$. albicans organisms adhered to fluoromonomer-added specimens tended to decreased with the carbon chain length of the fluorinated alkyl acrylate used as $\mathrm{co}^{-}$ monomer, although no statistical difference was obtained for the difference between C8FA and C10FA. In addition, Pearson's correlation coefficients between the contact angle for water and the number of $C$. albicans adhered to unpolished and polished specimens were similar $-0.977(p<0.01)$.

\section{Adherence of C. albicans to saliva-coated specimen surfaces}

Fig. 2. shows the numbers of $C$. albicans organisms adhered to saliva-coated specimens. The numbers found on saliva-coated C4FA, C6FA, C8FA, and C10FA were 19.4, 16.2, 12.4, and $10.3 \mathrm{cells} / \mathrm{mm}^{2}$, respectively, while that of the control was 53.2 cells/ $\mathrm{mm}^{2}$. The numbers of $C$. albicans organisms adhered to the specimens made using fluorinated alkyl

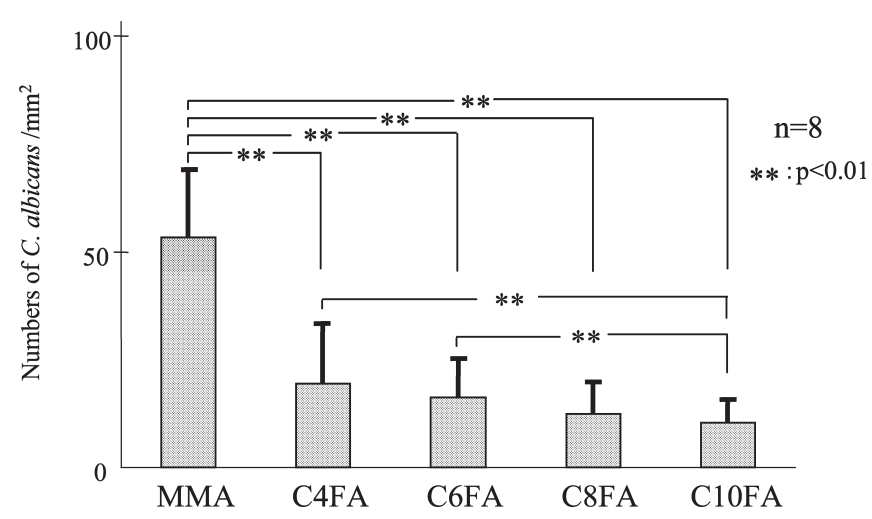

Fig. 2. Adherence of $C$. albicans to saliva-coated specimen acrylate were significantly lower than that found for the control. Furthermore, the number of C. albicans organisms adhered to C10FA was significantly lower than those found for C4FA and C6FA. Pearson's correlation coefficient between contact angle for water and the number of adhered C. albicans was $-0.996(p<0.01)$.

\section{Discussion}

Fluorine has a relatively small Van der Waals force and relatively large electronegativity. In addition, the $\mathrm{C}-\mathrm{F}$ bond possesses greater stability and strength than the $\mathrm{C}-\mathrm{H}$ bond (13). These properties give fluoro-materials heat resistance, low surface tension, high physiological activity, and superior heat, chemical, and weather resistance. The polyfluoroalkyl or polyfluoroalkylene group (RF group), particularly, is less likely to bend due to repulsion between the fluorine atoms, and its intermolecular interactions with other polar molecules are suppressed significantly because the $\mathrm{C}-\mathrm{F}$ bond distance is slightly smaller than the $\mathrm{C}-\mathrm{H}$ bond distance, allowing the fluorine atoms to completely surround the carbon chain (14). Therefore, rod-shaped RF groups aggregate in an orderly fashion. Because the surface energy of this aggregate is extremely low, the fluoroalkyl side chain orients toward the surface exposed to air of the resulting fluoropolymer.

In this study, we examined the influence of carbon chain length of fluorinated alkyl acrylate on the surface properties and bacterial adhesion to copolymers. All specimens with fluoromonomers exhibited significantly higher water- and oil-shedding surface characteristics compared to the control, as shown in Tables 1 and 2. This surface modification produces superior resistance to discoloration and plaque formation on the denture base in the oral cavity $(15,16)$. Furthermore, the contact angle for water and dodecane on the fluoromonomer-added specimens increased with carbon chain length of the perfluoroalkyl groups, although no statistical difference existed among the contact angles of C6FA, C8FA, and C10FA. A previous study (17) described the relation between hydrophobicity and $\mathrm{co}^{-}$poly- 
mers such as acrylate and fluorinated alkyl acrylate. When the carbon chain length of the fluorinated alkyl acrylate was less than eight, the static contact angles of the co-polymers were relatively low. The crystallization of the fluoroalkyl carbon chain affects the contact angles for water and dodecane.

The number of $C$. albicans that adhered to salivauncoated and -coated fluoromonomer-added specimens was significantly lower compared to the control (Figs. 1. and 2.). Furthermore, the numbers of $C$. albicans that adhered to fluoromonomer-added specimens decreased significantly with the carbon chain length of the perfluoroalkyl group, although no statistically significant difference existed between C8FA and C10FA. In contrast, the number of $C$. albicans that adhered to polished specimens were approximately $75 \%$ of that found for non-polished specimens. Surface characteristics of the mold affect the orientation of the fluoroalkyl side chain in the polymer during processing. Therefore, the surface of non-polished fluoromonomer-added specimens is relatively hydrophilic compared to the polished specimens.

Since the electronegativity of fluorine is high, the surface energy of an object decreases upon addition of fluorine atoms over the surface. Therefore, the surface of polymers containing fluorine is less likely to become wetted by a liquid with a high degree of intermolecular cohesion, is hydrophobic, and has a high contact angle for water. Ichimaru et al (18). reported that a greater contact angle reduced plaque adhesion and increased the ease of plaque removal. It has been reported that plaque is less likely to adhere to materials with low surface energy (16), and the number of $C$. albicans organisms adhered to fluoromonomer-added specimens decreased with carbon chain length of the perfluoroalkyl groups.

This study suggests that the addition of fluorinated alkyl acrylate to denture base resin increases the hydrophobicity of the surface and that fluorinated alkyl acrylates with relatively long carbon chains, such as $\mathrm{C} 8 \mathrm{~F}$ and $\mathrm{C} 10 \mathrm{~F}$, inhibit bacterial adherence to the resin surface.

\section{Acknowledgments}

This study was partially supported by a grant from the Ministry of Education, Culture, Sports, Science, and Technology to promote 2001-Multidisciplinary Research Projects (2001-2005) and a grant from the Ministry of Education, Culture, Sports, Science, and Technology to promote research between academia and industry.

\section{References}

1. Fujinami Y, Hayakawa I, Hirano S, and Watanabe I : Changes in Postural Control of Complete Denture Wearers after Receiving New Dentures - Gait and Body Sway-, Prosthodont Res Pract, 2: 11-19, 2003.

2. Okuda K : Denture Plaque and Oral Bacteria Possess Latent Pathogenicity for Elderly, J Jpn Prosthodont Soc, 43 : 636-639, 1999.

3. Yoneyama T, Hashimoto K, Fukuda H, Ishida M, Arai H, Sekizawa K, Yamada M, and Sasaki H : Oral Hygiene Reduces Respiratory Infections in Elderly Bed-bound Nursing Home Patients, Arch Gerontol Geriatr, 22 : 11-19, 1993.

4. Hayashi R, Kubota T, and Mega J : Application of Fluoroalkyl Acrylate Monomer for a Denture Base Material, Int J Oral-Med Sci, 1 (2) : 124-129, 2003.

5. Kubota T, Kobayashi M, Hayashi R, Ono A, and Mega $\mathrm{J}$ : Influence of Carbon Chain Length of Fluorinated Alkyl Acrylate on Mechanical Properties of Denture Base Resin, Int J Oral-Med Sci, 4 : 92-96, 2005.

6. Kim JW, Suh KD : Highly Monodisperse Crosslinked Polymethyl-methacrylate Microparticles by Dispersion Polymerization. Colloid Polym Sci, 277: 66-72, 1999.

7. Wakasugi Y: Preparation of Poly (methyl metha-

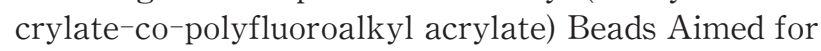
Denture Base Material. Dept. of Materials Science and Engineering, Ibaraki Univ, 2001. (master's thesis)

8. Hikita M, Tanaka K, Nakamura T, Kajiyama T, and Takahara A; Aggregation States and Surface Wetability in Films of Poly (styrene-block-2-perfluorooctyl ethyl acrylate) Diblock Copolymer Synthesized by Atom Transfer Radical Polymerization, Langmuir, 20: 5304-5310, 2000.

9. Otake S, Makimura M, Kuroki T, Nishihata Y, Hirasawa $\mathrm{M}$ : Anticaries effect of polyphenolic compounds from Japanese green tea, Caries Res, 25: 438-443, 1991.

10. Kohinata H: A Study of Adherence Mechanism of Candida albicans to Acrylic Resin or Human Buccal Epithelial Cells, Nihon Univ J Oral Sci, 21: 322-331, 1995.

11. Ishimura S: The Story of Analysis of Variance, 95- 
101, 136-149, 206-214, Tokyotosyo, Tokyo, Japan, 1992.

12. Nakai N, Matsubara N : Two-dimensional Data; in The Introduction of Statistics (Statistics Section, Department of Social Sciences, College of Arts and Sciences, The University of Tokyo), 41-54, The University of Tokyo, Tokyo, Japan, 1991.

13. Kitadume $\mathrm{T}$ : The Introduction ; in Fluoro Chemistry (Kitadume T, Ishihara T, Taguchi T), 1-4, Kodansya, Tokyo, Japan, 1993.

14. Ishikawa N : Properties, Functions and Compositions of Fluorocarbon; in Synthesis and Functions of Fluoromaterials (Ishikawa N), 1-7, CMC, Tokyo, Japan, 1987.

15. Kurosaka N, Nihei T, Hanaoka K, Kurata S, Sawada $\mathrm{T}$, Shimizu T, and Teranaka T : Surface Modification of Denture Base Resin by a Plasma Vapor Deposition
Polymerization Method, J Kanagawa Odont Soc, 36, 63-74, 2001.

16. Quirynen M, Marechal M, Busscher HJ, Weerkamp A H, Arends J, Darius PL, and van Steenberghe D: The Influence of Surface Free-energy on Planimetric Plaque Growth in Man, J Dent Res, 68 : 796-799, 1989. 17. Yamaguchi F : Functional Organofluorine Materials; in The Introduction of Fluorine Chemistry (Japan Society for the Promotion of Science The 155 th Committee on Fluorine Chemistry), 268-270, Sankyo, Tokyo, Japan, 2004.

18. Ichimaru T, Saito S, Matsuzaki A, Ando Y, and Furukawa K: Studies on Plaque Accumulation $(S$. mutans) in Various Dental Restorative Materials for Fluoro-resins (in vitro), J Jpn Dent Mat, 8: 337-348, 1989. 